\title{
IDENTIFICAÇÃO POR CG-EM E ANÁLISE COMPORTAMENTAL, VIA ACP, DE HPA E SUAS FONTES NO RIACHO ALGODOAIS - SUAPE/PE
}

\author{
D. P. SOUZA ${ }^{1}$, A.M.R.B. da SILVA ${ }^{2}$, J.C.SILVA ${ }^{1}$, R.V.L.SALES ${ }^{1}$, M. BENACHOUR ${ }^{1}$, \\ V.L. da SILVA ${ }^{1}$ \\ ${ }^{1}$ Universidade Federal de Pernambuco, Departamento de Engenharia Química \\ ${ }^{2}$ Universidade Federal de Pernambuco, Departamento de Engenharia Civil \\ E-mail para contato: daniellepiress@hotmail.com
}

\begin{abstract}
RESUMO - Os Contaminantes Emergentes (CE) têm contribuído no aumento da poluição ambiental, sobretudo em recursos hídricos, sendo estes caracterizados por se apresentarem em baixas concentrações, $\mu \mathrm{g} . \mathrm{L}^{-1}-\mathrm{pg} . \mathrm{L}^{-1}$, porém alta persistência e toxicidade. Dentre os $\mathrm{CE}$, os Hidrocarbonetos Policíclicos Aromáticos (HPA) são compostos orgânicos com dois ou mais anéis aromáticos conjugados, apolares, ubíquos, apresentando efeitos carcinogênicos e/ou mutagênicos para os seres vivos. No trabalho foi identificado e verificado o comportamento dos HPA e suas fontes no Riacho Algodoais, Suape-PE, onde realizou-se três coletas. Após extração líquido-líquido com diclorometano como solvente, as amostras foram injetadas no CG-EM (Cromatografia Gasosa acoplada a Espectrometria de Massa). Nove HPA foram identificados e quantificados. Para Análise de Componentes Principais utilizou-se o software Statistica8, onde o comportamento e as fontes dos HPA foram estudados. Razões diagnósticas entre o Fluoranteno e Pireno possibilitaram a identificação das fontes. Em duas coletas as fontes se classificaram como pirogênicas, havendo discordância na terceira coleta, onde a fonte determinada foi a petrogênica, tendo esse comportamento graças à presença do criseno e um aumento significativo na concentração do pireno encontrado. Faz-se necessário um monitoramento contínuo do Riacho Algodoais, pois a presença dos HPA em concentrações significativas representa uma ameaça aos seres vivos em geral e ao meio ambiente.
\end{abstract}

\section{INTRODUÇÃO}

A qualidade das águas tem sido objeto de preocupação visto a crescente poluição das mesmas. Dentre os poluentes, os Contaminantes Emergentes (CE) se caracterizam por sua persistência e toxicidade apesar de baixas concentrações, na ordem de $\mu \mathrm{g} . \mathrm{L}^{-1}-\mathrm{pg} . \mathrm{L}^{-1}$. Os estudos desses contaminantes tem se intensificado devido ao aumento de ocorrências em águas superficiais, alimentos e no ar e os efeitos ocasionados Por eles na saúde humana e dos seres vivos. Com o a avanço da química analítica, a detecção desses compostos em baixas concentrações possibilitam o conhecimento do seu comportamento, fontes e efeitos. (Ghiselli e Jardim (2007), Miranda - García et al., (2011), Soderstrom et al., (2009), Ternes (2010) e ASTDR (2009)). 


\section{9 a 22 de outubro de 2014 \\ Florianópolis/SC}

Em se tratando de CE, uma classe merece destaque: Hidrocarbonetos Policíclicos Aromáticos (HPA), compostos orgânicos com dois ou mais anéis aromáticos conjugados, formados durante a combustão incompleta ou pirólise de materiais contendo carbono e hidrogênio. São apolares, lipofílicos, considerados carcinogênicos, mutagênicos e/ou teratogênicos e consequentemente devido aos seus efeitos toxicológicos para os seres vivos em geral, sua facilidade de absorção e ubiquidade, pesquisas tendo como centro de interesse os HPA têm sido intensificadas a cada ano (Souza (2012), Wu, et al,.(2010), Rodil, et al., (2007),Pereira Netto, et al., (2000) e Cristale, et al., (2008)).

Os HPA são provenientes de duas fontes: naturais ou antropogênicas. Exemplos de fontes naturais são as atividades vulcânicas, afloramento de petróleo e sínteses biológicas. Das fontes antropogênicas podem ser citadas derramamento de petróleo, queima de combustíveis fósseis, queimadas de florestas, processos industriais e emissões automotivas. As fontes antropogênicas (ou antrópicas), são as principais responsáveis pela presença destes contaminantes no meio ambiente. (Lopes e Andrade (1996), Froehner e Martins (2008), Sodré et al., (2007), Sodré et al., (2010) e Cristale, et al. (2008)). Os HPA originários de fontes antropogênicas podem ser petrogênicos e pirogênicos, onde o primeiro é caracterizado pelos HPA de baixa massa molecular (BMM), sendo formados através de baixa temperatura de formação, podendo ser provenientes de derramamento de petróleo, combustão de matéria orgânica e lenta maturação da mesma. Os pirogênicos são aqueles que apresentam alta massa molecular (AMM), atribuídos a esse tipo de fonte estão, em grande parte, a combustão incompleta da madeira, óleo e carvão, a combustão incompleta de matéria orgânica a altas temperaturas, além de emissões veiculares. Para identificação pode-se utilizar uma razão entre o Fluoranteno (FL) e Pireno (P), FL/(FL + P). Quando for menor que 0,5: fontes petrogênicas; valores acima de 0,5; indicação de fontes pirogênicas (Pavei (2007); Silva (2004); Meniconi (2007), Souza (2012) e Veiga (2003)).

Para identificação, determinação e quantificação de CE do tipo HPA, é necessária a utilização de métodos analíticos que possuam baixos limites de detecção, alta eficiência e seletividade, que é o caso da Cromatografia Gasosa acoplada a Espectrometria de Massa (CG-EM). Para organização dos dados obtidos do CG-EM, uma técnica estatística que serve como auxiliar é a Análise de Componentes Principais (ACP), que se caracteriza pela organização dos dados, amostras e variáveis, analisadas em conjunto, visualizando assim os resultados dessas combinações. ACP é uma ferramenta eficaz para essas análises apresentando o mínimo de perda estatística (Faro Jr. et al., (2010) e Moura, et al.,(2006) e Beyer et al.,(2010).

O presente trabalho verificou o comportamento dos CE do tipo HPA através da ACP em amostras do Riacho Algodoais - Suape/PE. Este riacho recebe efluentes de indústrias dos mais variados tipos, sendo elas: bebidas, têxtil, fabricação de latas de alumínio, garrafas PET e produtos cerâmicos, além do esgoto doméstico que em sua maioria não recebe tratamento adequado. 


\section{MATERIAIS E MÉTODOS}

\subsection{Coleta}

Foram realizadas três coletas em períodos diferentes do ano em um mesmo ponto onde existe alta concentração de hidrocarbonetos policíclicos aromáticos (HPA), conforme resultados de pesquisas anteriores. O recurso hídrico escolhido, o Riacho Algodoais, é classificado pelo órgão ambiental de Pernambuco, CPRH, como água doce de classe 2, segundo o CONAMA 357/05. As amostras coletadas foram acondicionadas em vidrarias âmbar para evitar degradação das mesmas e refrigeradas $\mathrm{a}-4^{\circ} \mathrm{C}$.

\subsection{Análise por CG-EM}

A identificação e quantificação dos HPA foram realizadas via Cromatografia Gasosa acoplada a Espectrometria de Massa (CG-EM) da Shimadzu, modelo GCMS-QP2010 Plus, após extração líquido-líquido, com diclorometano p.a. (Merck) como solvente. O extrato obtido foi concentrado em um rota-evaporador a $40+1^{\circ} \mathrm{C}$. As amostras foram injetadas no CG-EM e uma curva de calibração na faixa de concentração, de 1 a $100 \mu \mathrm{g} . \mathrm{L}^{-1}$, foi utilizada para identificação e quantificação dos hidrocarbonetos.

\subsection{Análise via ACP}

Após a identificação e quantificação dos HPA presente na amostra, para a Análise de Componentes Principais (ACP), utilizou-se o software Statistica 8.0, onde foi possível uma análise comportamental dos HPA identificados e suas fontes.

\section{RESULTADOS}

Segundo Pinhati (2008), a Environmental Protection Agency (EPA) classificou com poluente prioritário para monitoramento ambiental 16 HPA devido a sua toxicidade, mutagenicidade e carcinogenicidade. Foram identificados nove HPA dos prioritários pela EPA, os quais apresentaram concentrações em nível de CE, ou seja, entre $\mu \mathrm{g} . \mathrm{L}^{-1}$ e pg. $\mathrm{L}^{-1}$. A Figura 1 apresenta as concentrações de oito destas substâncias nas três coletas. 


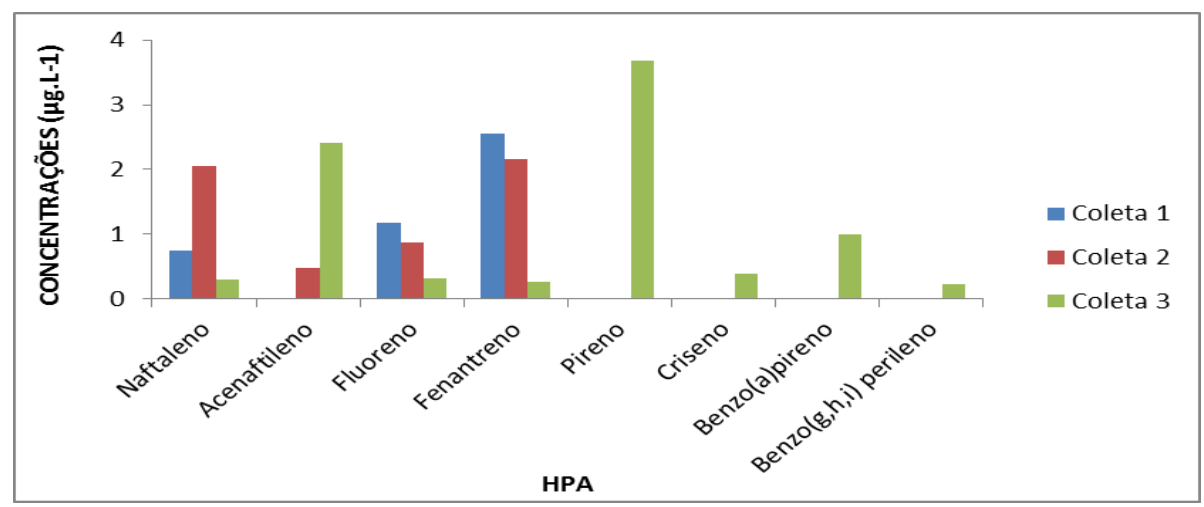

Figura 1- Concentrações de oito HPA nas três coletas do Riacho Algodoais

$\mathrm{Na}$ terceira coleta foram identificados quatro compostos que não haviam sido detectados nas duas primeiras. Todos esses composto são hidrocarbonetos de alta massa molecular (AMM), considerados mutagênicos e/ou teratogênicos, genotóxicos e carcinogênicos. O Benzo[a]pireno é considerado como um dos mais carcinogênicos, apresentando alta periculosidade e difícil degradação. O Fluoranteno também foi identificado nas três coletas. Os valores de suas concentrações podem ser observados na Figura 2, pois as mesmas apresentaram grande discrepância em relação às demais.

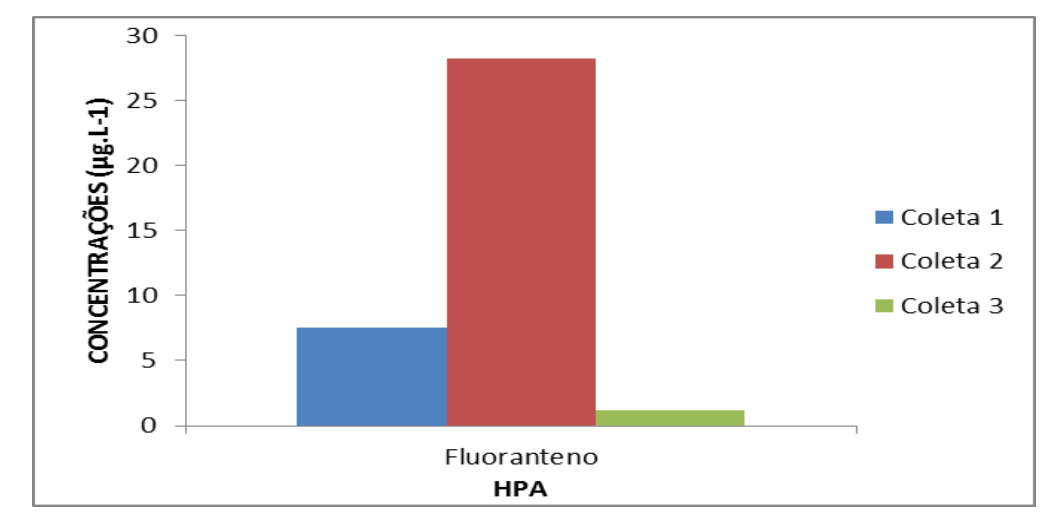

Figura 2 - Concentrações do Fluoranteno nas três coletas do Riacho Algodoais

O Fluoranteno é um HPA de AMM, não carcinogênico, porém genotóxico e ao apresentar altas concentrações, sugere-se como fontes processos em altas temperaturas.

Para determinação das fontes de HPA, foi realizado o cálculo das razões anteriormente mencionadas Na Tabela 1 vemos o resultado do cálculo dessa razão para as amostras do Riacho Algodoais. 
Tabela1 - Razão Diagnóstica para identificação das fontes das 3coletas

\begin{tabular}{|c|c|c|c|c|c|}
\hline \multicolumn{2}{|c|}{ COLETA 1 } & \multicolumn{2}{c|}{ COLETA 2 COLETA 3 } \\
\hline FL/FL+Pi & FONTE & FL/FL+Pi & FONTE & FL/FL+Pi & FONTE \\
\hline 1,00 & Pirogênica & 1,00 & Pirogênica & 0,24 & Petrogênica \\
\hline
\end{tabular}

Conforme observado na Tabela 1 , nas coletas 1 e 2 houve uma concordância nas fontes dos HPA, porém na terceira coleta a mesma se caracterizou como fonte petrogênica. Atribuiu-se a essa divergência a presença de HPA de origem mista, sendo um deles o pireno, segundo Cordeiro (2003), com um valor maior do que nas outras amostras e o criseno, anteriormente não detectados.

Para Análise de Componentes Principais, os valores encontrados das concentrações foram tratados no Statistica 8.0, onde se obteve uma matriz total dos dados de três amostras e nove variáveis. A Figura 3 apresenta os escores das amostras na CP1, caracterizando 86,54 \% das amostras de acordo com os HPA detectados. As coletas 1 e 2 se comportaram de maneira semelhante, verificando-se uma concordância no que diz respeito ao compostos identificados em ambas as coletas. No entanto, a coleta 3 apresentou-se de forma oposta as outras duas o que se atribui aos novos compostos identificados.

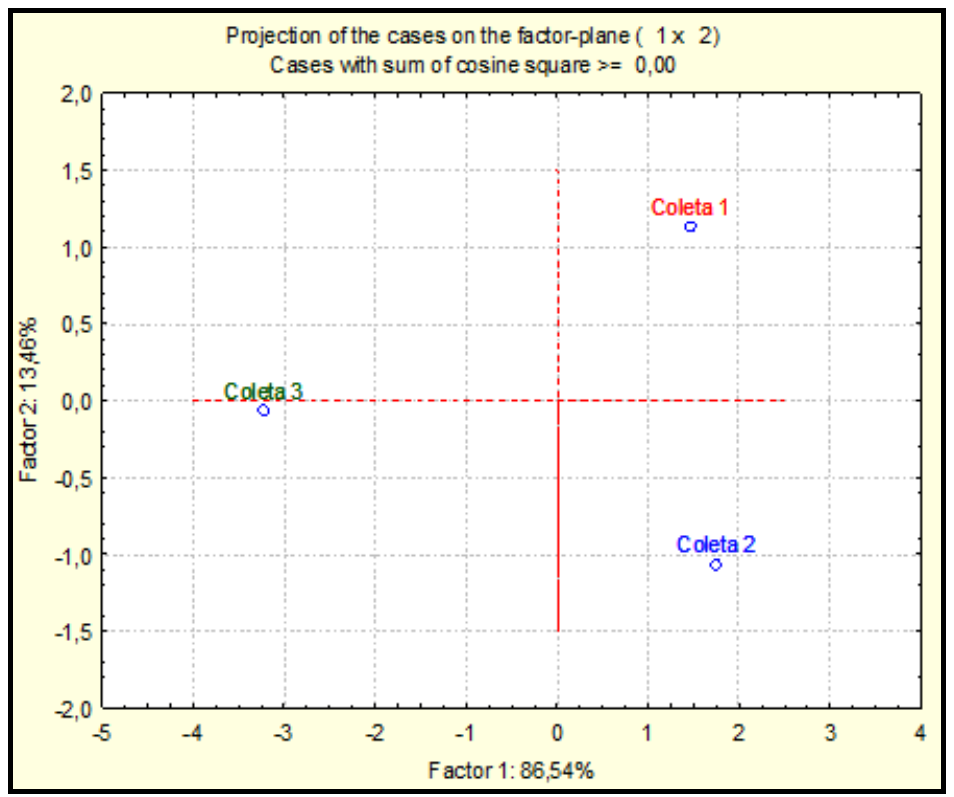

Figura 3- Gráfico das amostras das CP1 das coletas do Riacho Algodoais. 
A Figura 4 apresenta quais os HPA de maiores influências nas respectivas coletas.

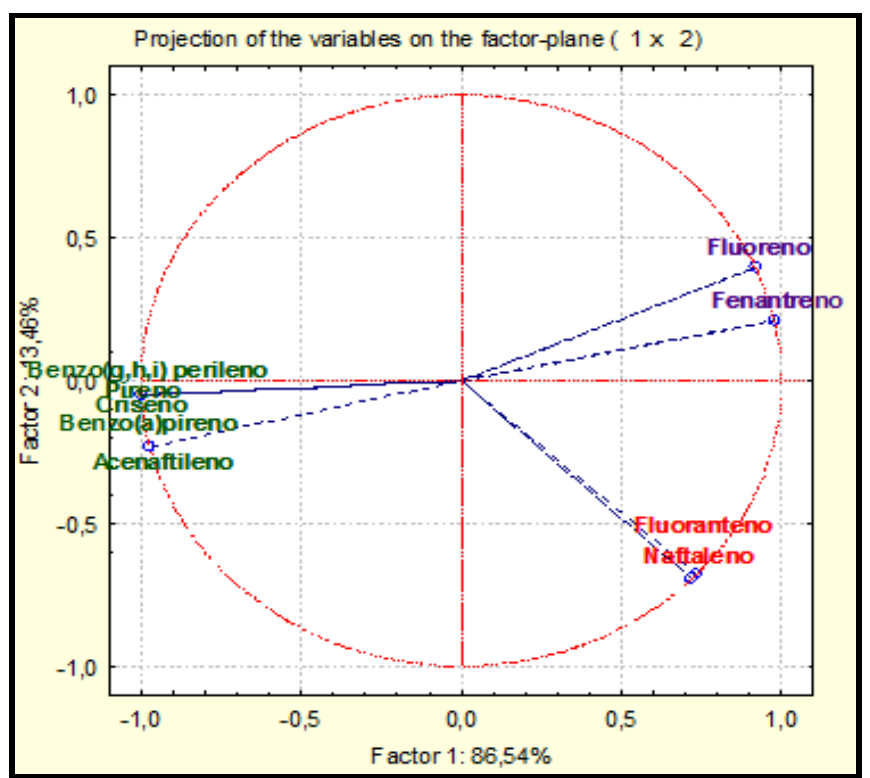

Figura 4 - Gráfico dos HPA das três coletas no Riacho Algodoais

A coleta 1 e 2 são influenciadas pelo mesmo conjunto de HPA, sendo assim de um mesmo tipo de fonte, a pirogênica. A coleta 3, por conseguinte, apresentou influência de outros HPA, sugerindo assim uma fonte diferente, petrogênica, comprovada pela razão diagnóstica anteriormente mencionada. Vale ressaltar que o Pireno e o Criseno, apresentado apenas na $3^{\text {a }}$ coleta, são compostos de origem mista.

\section{CONCLUSÃO}

A presença de contaminantes emergentes do tipo HPA se mostrou em quantidades significativas e preocupantes, ao longo de todo o riacho, visto os efeitos que esses micropoluentes causam nos seres vivos em geral. Nove dos dezesseis HPA prioritários para estudo ambiental pela USEPA (United States Environmental Protection Agency) foram identificados;

A Análise de Componentes Principais mostrou-se eficiente na visualização da concentração desses micropoluentes em cada coleta e quais apresentaram uma maior influência nas mesmas. Esta ferramenta é útil, pois os dados são reduzidos através de combinações lineares dos mesmos com características semelhantes;

A razão entre o Fluoranteno e o Pireno possibilitou a identificação e confirmação das fontes de contaminação desses HPA nas três coletas. Nas duas primeiras houve uma concordância em fontes 
pirogênicas, porém na $3^{\text {a }}$ coleta houve uma mudança e no ponto 2 , onde se identificou uma influência mais significativa de fontes de origem petrogênicas. Esse comportamento pode ser justificado pelo Pireno, que se apresentou com um valor mais significativo e o Criseno anteriormente não detectado, sendo esses compostos de origem mista; São necessários maiores estudos, pois foi possível constatar uma contaminação persistente desses micropoluentes ambientais.

É necessário um monitoramento contínuo deste Riacho, devido às concentrações significativas dos HPA e os efeitos tóxicos nos animais e no meio ambiente que eles representam.

\section{REFERÊNCIAS}

ASTDR, Agency for Toxic Substances and Disease Registry. Case Etudies in Environmentam Medicine Toxicity of Polycyclic Aromatic Hydrocarbons (PAH). Course: WB, 1519, 2009.

BEYER, J.; JONSSON, G.; PORTE, C.; KRAHN, M.M.; ARIESE, F. Analytical methods for determining metabolites of polycyclic aromatic hydrocarbon (PAH) pollutants in fish bile: A review. Environmental Toxicology and Pharmacology, v. 30, pp. 224-244, 2010.

CORDEIRO, L. H. Hidrocarbonetos Policíclicos Aromáticos nos Sedimentos do Estuário Da Laguna Dos Patos - RS. Dissertação de Mestrado. Rio Grande- RS: Universidade Federal de Rio Grande, 2003.

CRISTALE, J.; SILVA, F. S.; MARCHI, M. R. R. "Desenvolvimento e Aplicação de Método GCMS/ MS para Análise Simultânea de 17 HPAs em Material Particulado Atmosférico". Eclética Química, vol.03, nº , pp. 69-78, 2008.

FARO Jr. A.C.; RODRIGUES, V.O.; EON, J.; ROCHA, A.S. Análise por componentes principais de espectros nexafs na especiação do Molibdênio em catalisadores de hidrotratamento. Química Nova, v. 33, no 6, pp. 1342-1347, 2010.

FROEHNER, S.; MARTINS, R.F. Avaliação do destino e bioacumulação de benzo(a)pireno através de simulação computacional. Química Nova, v.31, nº5, p. 1089-1093, 2008.

GHISELLI, G.; JARDIM, W.F. Interferentes Endócrinos no Ambiente. Química Nova, v. 30, nº 3, 695-706, 2007.

LOPES, W.A.; ANDRADE, J.B. Fontes, Formação, Reatividade e Quantificação de Hidrocarbonetos Policíclicos Aromáticos (HPA) na Atmosfera. Química Nova, v. 19, 497-516, 1996.

MENICONI, M.F.G. Hidrocarbonetos Policíclicos Aromáticos no Meio Ambiente: Diferenciação de Fontes em Sedimentos e Metábolitos em Biles de Peixes. Tese de Doutorado. UFRN, Natal, RN, 2007.

MIRANDA-GARCÍA, N.; SUÁREZ, S.; SÁNCHEZ, B.; CORONADO, J.M.; MALATO, S.; IGNACIO MALDONADO, M. Photocatalytic degradation of emerging contaminants in municipal 
wastewater treatment plant effluents immobilized $\mathrm{TiO} 2$ in a solar pilot plant. Applied Catalysis B: Environmental, v. 103, p. 294-301, 2011.

MOURA, M.C.S.; LOPES, A.N.C.; MOITA, G.C.; MOITA NETO, J.M. Estudo multivariado de solos urbanos da cidade de Teresina. Química Nova, v. 29, n 3, pp.429-435, 2006.

PAVEI, P.T. Caracterização e estudo do comportamento de Hidrocarbonetos Policíclicos Aromáticos em ecossistemas aquáticos contaminados pelas atividades de mineração de carvão. Dissertação de Mestrado. Criciúma- SC: Universidade do Extremo Sul Catarinense, 2007.

PEREIRA NETTO, A.D.; MOREIRA, J.C.; DIAS, A.E.X.O.; ARBILLA, G.; FERREIRA, L.F.V.; OLIVEIRA, A. S.; BAREK, J. Avaliação da Contaminação Humana por Hidrocarbonetos Policíclicos Aromáticos (HPAs) e seus Derivados Nitrados(NHPAS): Uma Revisão Metodológica. Química Nova, V. 23, nº 6 , pp.765-773, 2000.

RODIL, R.; SCHELLIN, M.; POPP, P. Analysys of Polycyclic Aromatic Hydrocarbons in water and beverages using membrane- assisted solvent extraction in cvombination with large volume injectiongas chromatography - mass spectrometric detection. Journal of Chormatography A, v. 1163, p. 288297, 2007.

SILVA, G.S. Avaliação do Estado de Degradação e Capacidade de Suporte da Bacia do Rio AtibaiaRegião de Campinas/ Paulínia - SP. Tese de Doutorado. UNICAMP, Campinas, SP, 2004.

SÖDERSTRÖM, H.; LINDBERG, R.H.; FICK, J. Strategies for monitoring the emerging polar organic contaminants in water with emphasis on integrative passive sampling. Journal of Chromatography A, v. 1216, p. 623-630, 2009.

SODRÉ, F.F.; LOCATELLI, A.F.; JARDIM, W.F. Sistema Limpo Em Linha Para Extração Em Fase Sólida De Contaminantes Emergentes Em Águas Naturais. Química Nova, v. 33, nº 1, pp. 216/219, 2010.

SODRÉ, F.F.; LOCATELLI, M.A.F.; MONTAGNER, C.C.; JARDIM, W.F. Origem e Destino de Interferentes Endócrinos em Águas Naturais. Caderno Temático, Campinas, SP: UNICAMP, v.6, 2007.

SOUZA, D.P. Avaliação de Contaminantes Emergentes do tipo HPA no Riacho Algodoais Suape-PE e Tratamento via Processo Oxidativo Avançado. Dissertação de Mestrado. Recife, PE: Universidade Federal de Pernambuco, 2012.

TERNES, T. Editorial to special issue in Water Research Energing contaminants in water. Water Research, v.44, pp. 351, 2010.

VEIGA, I.G., Avaliação da Origem dos Hidrocarbonetos em Sedimentos Superficiais de Manguezais da Região Norte da Baía de Todos os Santos/Bahia. Dissertação de Mestrado. Macaé, RJ: UENF, 2003.

WU, J.; HOU, H.; RITZ, B.; CHEN, Y. Exposure Polyciclic Aromatic Hydrocarbons and missed abortion in early pregnancy in a Chinese population. Science of the Total Environment, v. 408, pp. 2312-2318, 2010. 